\title{
BEM-ESTAR SUBJETIVO E DESEMPENHO ACADÊMICO EM ESTUDANTES UNIVERSITÁRIOS
}

Ana Paula Domeneghetti Parizoto Fabrin, Camélia Murgo Mansao

Universidade do Oeste Paulista - UNOESTE. Faculdade de Psicologia, Presidente Prudente-SP.

\section{RESUMO}

Este estudo teve como objetivo verificar correlações entre desempenho acadêmico e bem-estar subjetivo, sob a perspectiva da Psicologia Positiva, que contribui com definições do construto "Bem-Estar subjetivo", suas implicações no desempenho acadêmico, e nas crenças de autoeficácia profissional. Comparações por gênero foram levantadas neste estudo. Foi formada uma amostra de alunos de dois cursos de uma Universidade do interior paulista, cujos instrumentos de medida contemplaram a Escala de Afetos Positivos e Negativos Zanon (EAZ) e a verificação do desempenho acadêmico pelas médias globais de dois semestres. Os resultados apontaram que não foram encontradas correlações entre as médias de notas dos estudantes universitários com os afetos positivos e negativos, componentes do bem-estar subjetivo avaliados pela EAZ, contrapondo as hipóteses iniciais da pesquisa. Já as notas se correlacionaram entre si. Também foi apontada inexistência de diferenças entre as médias, estatisticamente, em relação a gênero.

Palavras-chave: Bem-Estar Subjetivo. Desempenho acadêmico. Psicologia Positiva. Escala de BemEstar.

\begin{abstract}
This study aimed to examine correlations between academic performance and subjective wellbeing from the perspective of Positive Psychology, which helps with definitions of the construct "Subjective Well-Being", its implications on academic performance, and professional self-efficacy beliefs. Comparisons by gender were raised in this study. A sample of students from two courses of University of São Paulo, whose measuring instruments contemplated Scale Positive and Negative Affects Zanon (EAZ) and the verification of academic performance the global averages of two semesters was formed. The results showed that no correlations between mean scores of college students with positive and negative affect components of subjective well-being assessed by EAZ were found, comparing the initial hypotheses of the research. Already notes correlated with each other. Was also indicated no differences between the means, statistically, in relation to gender.
\end{abstract}

Keywords: Subjective Well-Being. Academic performance. Positive Psychology. Scale Welfare. 


\section{INTRODUÇÃO}

A Orientação de Carreira pode ser contemplada desde o ensino universitário até posteriormente, durante a fase produtiva e profissional do indivíduo, favorecendo a saúde mental no trabalho e a consequente realização pessoal e profissional. A perspectiva da Psicologia Positiva, através das definições do construto bem-estar subjetivo, suas implicações no desempenho acadêmico e nas crenças de autoeficácia profissional, tem contribuído para o entendimento das possíveis associações entre carreira, felicidade e desempenho, de acordo com trabalhos realizados por Ramos e Ramos (2004) e também por Noronha e Mansão (2012).

A Psicologia Positiva foi definida por Seligman (1999) e citado em Contreras e Esguerra (2006) como sendo um estudo, baseado na metodologia científica, de experiências positivas individuais, e realizado por instituições que facilitam a melhoria de qualidade de vida, evitando ou reduzindo a incidência de psicopatologia. É o estudo das forças e virtudes humanas, abrindo margem ao pleno potencial humano, suas motivações e habilidades, favorecendo a instauração e manutenção da saúde mental dos indivíduos.

Segundo Ramos e Ramos (2004), entende-se por Psicologia Positiva, a abordagem que visa trazer à reflexão a totalidade do ser humano na teoria e prática psicológicas. Esta vem mudar a direção da ciência psicológica, e ao invés de encampar somente a vertente dos problemas de natureza psicológica e o prisma das dificuldades e distúrbios, volta-se também para os valores subjetivos, as aspirações e o bem-estar. Provindo deste paradigma, a Psicologia não se limita apenas ao estudo, interpretação e superação das patologias, ou transtornos comportamentais, mas também a aspectos que caracterizam as virtudes, como o vigor $\mathrm{e}$ as potencialidades. A intervenção psicológica não se limitará a trabalhar as questões "erradas" no individuo, mas sim estimulará e motivará o que é suscetível de ser enriquecido. Esse enfoque permitirá re-orientar a Psicologia conforme novas perspectivas, tais como as de motivar o autofortalecimento, estimulando a produtividade das pessoas, a fim de alcançar um real sucesso em suas ações.

Os construtos derivados da Psicologia Positiva incluem o otimismo, a esperança, auto-eficácia e felicidade. Os vértices em que se constroem os procedimentos de avaliação apóiam-se desde o cognitivo, o emocional, o interpessoal, o religioso, social, entre outros, abrindo-se com isso a possibilidade de diálogo entre outras abordagens como a Psicologia Cognitiva, e a Psicologia Existencial (RAMOS; RAMOS, 2004).

Especificamente, acerca do bem-estar subjetivo, a Psicologia Positiva afirma que o 
conceito está associado com um envelhecimento saudável e pode ser entendido como sinônimo de felicidade, força do ego, ajuste, e integração social. À medida que as pessoas se percebem com recursos satisfatórios para lidar com fatores estressantes, aliados às sensações de prazer e alegria, ocorrem os chamados "fatores de proteção", que são, basicamente, competências e habilidades pessoais de saúde. Dentre os componentes do bem-estar subjetivo estão a satisfação com a vida e o equilíbrio dos afetos positivos e negativos, incluindo a noção de evolução cognitiva, qualidade de vida e relações afetivas.

O que compõem os fatores preditivos do bem-estar subjetivo são as relações afetivas, lazer, transporte e remuneração, o autoconceito de futuro e trabalho, matrimônio, relações interpessoais, aspectos que dependem da cultura e da maneira que se estruturam a vida de cada um; a percepção de autoeficácia, satisfação com a própria vida e felicidade (NORIEGA; QUIÑONES; GUEDEA, 2005).

Neri (2002) afirma ainda que o bemestar também inclui a noção de evolução cognitiva, qualidade de vida e relações afetivas, justapondo aos fatores objetivos, estariam os conceitos subjetivos e construídos ao longo do ciclo vital. Também coloca que o bem-estar subjetivo não diminui com a idade, apesar do declínio em saúde, estado civil e renda no idoso (NERI, 2002).

Dentre as pesquisas que já foram realizadas abordando as questões da psicologia positiva encontram-se os trabalhos de Londoño (2009) que desenvolveu uma pesquisa avaliando o grau de otimismo e saúde positiva das quais podem afetar a adaptação à vida acadêmica de estudantes universitários. A referida pesquisa foi realizada sobre uma amostra de 77 estudantes do gênero feminino e masculino, cuja idade variou entre 17 e 26 anos, correspondendo aos ingressantes do primeiro semestre acadêmico em 2005 do curso diurno de Psicologia de uma universidade privada. Os instrumentos utilizados foram o Teste de Orientação Vital (LOT), a Escala de Otimismo Seligman, Questionário de Saúde Mental Positiva, Escala de Satisfação Geral e uma ficha de registro de informação acadêmica, aplicados no ingresso ao curso de graduação, e posteriormente, no ano subseqüente. Como resultado, foi comprovada a correlação $(p<$ 0.05) entre o grau de otimismo e, inversamente proporcional, a desistência dos alunos no curso. Consequentemente foi possível utilizar o otimismo como um fator preditivo no cancelamento e repetição das disciplinas curriculares. Tanto o cancelamento como a repetição são sinais de risco para o abandono ou impossibilidade de 
conclusão do referido curso (LONDOÑO PÉREZ, 2009).

Outra pesquisa realizada por Martinez (2007) mostrou que há correlações entre grau de otimismo de analistas de mercado de capitais de empresas brasileiras com 0 desempenho de precisão e acurácia nas projeções dos analistas. Encontraram um viés de seleção otimista nas previsões dos analistas, e a pesquisa termina propondo uma metodologia cujo objetivo é ajustar as previsões de consenso de modo a torná-las mais acuradas que podem implicar naqueles que levam em consideração as previsões de resultados dos analistas nos modelos de avaliação de empresas. A precisão das projeções de resultados foi analisada através do banco de dados do sistema I/B/E/S, base esta que tem sido o mais importante fornecedor de previsões de lucros para profissionais de investimentos em todo o mundo. O período analisado compreendeu os anos de 1996 e 2002. O erro de previsão foi calculado pela diferença entre o resultado real (efetivo) e o resultado estimado pelos analistas.

Estes estudos permitiram concluir que as previsões dos analistas são otimistas em termos médios, sendo este considerado como viés de seleção. No viés de seleção presume-se que cada analista revele sua verdadeira expectativa em relação ao desempenho da firma. Entretanto, aqueles analistas que acreditam que a firma terá um desempenho pobre, optam por não liberar suas estimativas, aumentando assim o grau de sucesso nas análises médias restantes, mostrando uma previsão distorcida, portanto (MARTINEZ, 2007).

Como mais um exemplo, o trabalho de Dela Coleta e Dela Coleta (2006) mostrou uma correlação múltipla e positiva entre o bem-estar subjetivo e o comportamento acadêmico-universitário. A investigação envolveu 252 estudantes universitários dos últimos períodos de diversos cursos $\mathrm{e}$ diferentes instituições de educação superior. As respostas dos alunos envolviam perguntas sobre dados biográficos, escalas sobre o sentimento de felicidade, satisfação e bemestar subjetivo, avaliações de vários aspectos de suas vidas pessoais e acadêmicas. A análise destes estudos sugeriu que a realização acadêmica faz parte dos fatores de felicidade na opinião dos sujeitos brasileiros e que a educação é considerada um preocupante problema social.

Por fim, Dela Coleta \& Dela Coleta (2006) concluíram que os sentimentos estudados pela Psicologia Positiva estão mesmo relacionados com a conduta acadêmica, demonstrando-se que os acontecimentos nesta área particular estão associados a uma vida psíquica rica e saudável entre os estudantes universitários. Afirmam, ainda, que mudanças planejadas ao 
estilo e características da vida dos universitários podem influenciar os níveis de bem-estar e felicidade experimentados determinando, desta forma, as condutas acadêmicas mais desejáveis.

Outra pesquisa, de Gondim et.al. (2009) abordou os impactos de fatores contextuais na atribuição de afetos a um supervisor e um empregado interagindo em uma situação rotineira de trabalho. Participaram do estudo 465 estudantes universitários de Salvador e Madri. O objetivo foi apresentar uma análise qualitativa das respostas a uma questão aberta em que se pedia ao participante da pesquisa que se colocasse no lugar do supervisor e do empregado atribuindo afetos a cada um dos atores. Uma das conclusões desta pesquisa, é que expectativas sociais nas relações de trabalho são bastante ambivalentes e contraditórias, desencadeando, ao mesmo tempo, afetos positivos e negativos em relação ao mesmo ator, do mesmo modo que as sociabilidades positiva e negativa na interação não são interpretados de modo linear (GONDIM et.al., 2009). Isso mostra a relevância dos afetos positivos e negativos nas relações interpessoais dentro do contexto profissional, e sua implicação na saúde mental dos trabalhadores.

As emoções e os afetos também determinam a direção das decisões de gestão e de estratégia da empresa (GARCIA; SABATÉ;
MAZAGATOS, 2008), evidenciando a necessidade de estudos nas características positivas e negativas dos indivíduos e sua consequente repercussão nas realizações profissionais.

Se levados em consideração os achados teóricos acerca do bem-estar subjetivo, justifica-se a preocupação do presente estudo em buscar investigar índices de satisfação, felicidade, gratidão, deleite, em amostras de estudantes universitários, e sua relação com o comportamento acadêmico, até como forma de submeter a teste, ao se considerarem as atividades escolares, a conclusão de que níveis mais altos de felicidade e satisfação estariam relacionados a comportamentos mais positivos no trabalho (SELIGMAN, 2004).

Assim, como objetivos do presente trabalho, foram feitas as verificações dos níveis de bem estar subjetivo e as possíveis correlações com desempenho acadêmico de universitários sendo também investigadas as possíveis diferenças nos escores de bem estar entre os diferentes grupos etários e gênero.

\section{MÉTODO}

\section{Participantes}

A amostra foi composta por 131 alunos dos 60 e 8 termos do curso de Ciência da Computação e Psicologia da universidade na qual foi realizada a pesquisa. 


\section{Instrumentos}

Dentre os instrumentos, foram utilizados a Escala de Afetos Positivos e Negativos Zanon (EAZ) - que constitui-se de uma escala Likert de 5 pontos, variando de 'nada a ver com você' (1 ponto) a 'tudo a ver com você' (5 pontos), buscando avaliar os afetos positivos e negativos. A escala é composta por 20 afirmações que descrevem sentimentos e emoções passadas e presentes, como por exemplo, "Sou apaixonado por algumas coisas que eu faço". O instrumento é autoaplicável, sendo necessária apenas a folha de resposta, a qual contém a descrição dos itens.

Sua construção partiu de um estudo inicial, no qual 853 universitários (57\% mulheres), com média de idade de 21 anos $(D P=3)$, responderam a 29 itens, constantes na primeira versão do instrumento, os quais foram submetidos às Análises de Componentes Principais, por rotação Oblimin. Não foram encontrados índices satisfatórios de consistências interna para o modelo de três e quatro fatores, além de apresentarem diversos itens com cargas fatoriais acima de 0,35 em mais de um fator, e não fazerem sentido teoricamente. Portanto, não foi considerada pertinente essa distribuição.

Em uma análise bifatorial, foram eliminados os itens que apresentaram cargas maiores que 0,35 em mais de um fator ou comunalidades menores que um. Foram mantidos aqueles com índices fatoriais variando de 0,38-0,77. Assim, a versão final da EAZ passou a conter 20 afirmativas, sendo 10 referentes à subescala de afetos positivos e 10 , à de afetos negativos.

Os resultados da análise componencial mostraram que o índice Kaiser-Meyer-Olkin foi de 0,90 e o teste de esfericidade de Bartlett foi significativo $(p<0,001)$. A solução bifatorial produziu o primeiro fator com eigenvalue de 5,1 que explicou $25,6 \%$ da variância total e o segundo fator com eigenvalue de 2,6 que explicou 12,8\% da variância total. A consistência interna da escala, avaliada através do alfa de Cronbach, foi de 0,83 para afeto positivo e 0,77 para afeto negativo. As evidências de validade foram buscadas por meio das correlações entre a EAZ e a Positive and Negative Affect Schedule (PANAS). Observaram-se índices altos de correlação entre os afetos positivos $(0,73)$ e afetos negativos $(0,74)$, o que indica que as subescalas da EAZ medem o mesmo construto das subescalas da PANAS.

Outro instrumento foi a Verificação do Desempenho Acadêmico - Levantamento da média geral do aluno nos últimos dois semestres do ano vigente da coleta dos dados, totalizando duas médias semestrais para cada aluno participante, a saber o 
segundo semestre de 2011 e o primeiro semestre de 2012.

\section{Procedimentos}

O desenvolvimento do estudo teve inicio após a aprovação do projeto pelo Comitê de Ética em Pesquisa (Protocolo n. 1079). A coleta de dados foi realizada com a anuência dos coordenadores dos cursos de Informática e Psicologia durante o horário de intervalo das atividades acadêmicas. O Termo de Consentimento esclarecido foi encaminhado aos participantes voluntários, que assinaram o mesmo e responderam aos itens de bem estar subjetivo. Todo o material, bem como o Termo de Consentimento, foi recolhido pelos pesquisadores, sendo a aplicação do instrumento feita de forma coletiva.

\section{RESULTADOS}

Dentre as análises feitas, foi levantada a correlação entre a escala Zanon de afetos e o desempenho acadêmico, mensurado pelas médias de dois semestres consecutivos dos universitários. Assim, estabeleceu-se o levantamento da correlação entre os afetos positivos e negativos, com as médias finais do último semestre de 2011 e o primeiro semestre de 2012. A universidade onde ocorreu o estudo segue o modelo semestral de disciplinas, o que confere uma média global ao final de cada semestre do ano. Foi esta a média utilizada no estudo estatístico. Considerando a tabela abaixo, a escala de afetos, em ambos os fatores, tanto positivo como negativo, não se correlacionou com nenhuma das médias de notas (desempenho acadêmico). Já as notas se correlacionaram entre si $(r=0,76, p<0,001)$.

Tabela 01. Correlação entre a escala de afetos e as médias de notas

\begin{tabular}{lcccc}
\hline & Afetos positivos & Afetos negativos & media.2.2011 & media.1.2012 \\
\hline Afetos positivos & 1,00 & & & \\
Afetos negativos & 0,14 & & & \\
media.2.2011 & 0,03 & 0,09 & 1,00 & \\
media.1.2012 & $-0,01$ & 0,04 & $0,76^{*}$ & 1,00 \\
\hline
\end{tabular}

$* p<0,001$

Outra análise feita foi a diferença de média entre os gêneros. Foi verificado se haveria diferença entre os sexos na pontuação da escala de afetos, em ambos os fatores (positivo e negativo). Conforme tabela abaixo (tabela 02), as médias não foram consideradas diferentes estatisticamente. 
Tabela 02. Diferença de média entre sexo

\begin{tabular}{lccccc}
\hline & Média & Dimensão & $\mathrm{t}$ & $\mathrm{gl}$ & $\mathrm{p}$ \\
\hline Feminino & 38,13 & Afeto positivo & 0,860 & 118,051 & 0,391 \\
Masculino & 36,84 & & & & \\
\hline Feminino & 25,00 & Afeto negativo & $-1,231$ & 115,225 & 0,220 \\
Masculino & 26,83 & & & & \\
\hline
\end{tabular}

*São consideradas diferenças significativas aquelas em que $p<0,05$

\section{DISCUSSÃO}

Como hipótese inicial foi levantada a possibilidade de haver uma possível correlação entre os afetos positivos e um melhor aproveitamento acadêmico. Esta hipótese se baseou em várias pesquisas já realizadas, com resultados no mesmo sentido apontado, haja vista as pesquisas aqui citadas, como Ramos e Ramos (2004); Noronha e Mansão (2012), onde encontraram correlações entre a evolução na carreira e o grau de felicidade, um dos itens do bem-estar subjetivo. Londoño Pérez (2009) também encontrou correlação entre o grau de otimismo e saúde positiva; Martinez (2007) mostrou correlação entre o grau de otimismo de analistas de mercado e desempenho na previsibilidade e acurácia nas projeções dos mesmos. Entretanto, Martinez (2007) também ressaltou haver vieses nos dados obtidos. Gondim et. al. (2009) e também García, Sabaté e Mazagatos (2008) pesquisaram e demonstraram impactos de fatores contextuais entre afetos positivos e relações no trabalho. Assim sendo, haveria por hipótese uma busca por alguma correlação entre afetos positivos e possível aumento no desempenho acadêmico, algo que não tinha sido pesquisado desde então.

Dela Coleta e Dela Coleta (2006) mostrou correlação múltipla e positiva entre o bem-estar subjetivo e o comportamento acadêmico universitário, mas não chegaram a estudar pela escala de afetos de Zanon. Contrapondo este resultado, no presente estudo, pelos resultados obtidos e utilizandose da escala Zanon de Afetos positivos e negativos, a hipótese levantada foi refutada, uma vez que não houve correlação encontrada entre médias acadêmicas e afetos, em ambos os fatores, tanto positivo como negativo. Também não houve correlação ou diferenças entre os gêneros estatisticamente.

Monteiro, Tavares e Pereira (2008) também encontraram correlações positivas entre o otimismo disposicional e o bem-estar subjetivo, e ainda, também encontraram correlações entre o bem-estar e rendimento acadêmico em estudantes do primeiro ano do ensino superior.

Contrapondo a pesquisa de Monteiro; Tavares e Pereira (2008) e Dela Coleta e Dela 
Coleta (2006), na presente pesquisa, não foi encontrado correlação entre bem-estar e desempenho acadêmico, embora o instrumento utilizado tenha sido diferente das pesquisas aqui citadas.

Com relação à diferença envolvendo gêneros, houve um estudo de Giacomoni (2002), envolvendo vários levantamentos e constatações de correlações envolvendo diferentes itens de bem-estar com gênero, como mania, grau de raiva, excitabilidade, felicidade, otimismo. Giacomoni (2002) constatou diferenças entre os gêneros. Diferentemente, neste presente estudo, não houve diferenças significativas, estatisticamente, entre gêneros e desempenho acadêmico, e não houve diferenças entre bem-estar subjetivo nos diferentes sexos. Corroborando com estes resultados, a pesquisa de Queroz e Neri (2005) envolvendo bem-estar psicológico e Inteligência Emocional entre homens e mulheres de meia-idade e velhice, mostrou correlação entre competências relacionadas ao autodesenvolvimento e às relações interpessoais, mostrou em adição que bemestar psicológico e inteligência emocional não se comportaram como construtos independentes, e nem foram consistentemente afetados por gênero e idade, o que foi confirmado no presente trabalho.
Otta e Fiquer (2004) fizeram um estudo para investigar o sentimento de bemestar em função da idade e gênero. Obtiveram o resultado de que idosos de forma geral consideraram-se mais satisfeitos com a vida em relação a pessoas mais jovens. Homens jovens avaliaram-se como mais calmos do que mulheres de mesma idade. Estes resultados não foram constatados no presente trabalho, que não encontrou diferenças entre gêneros. A pesquisa de Otta e Fiquer (2004) envolveu 81 homens e 84 mulheres.

\section{CONCLUSÃO}

Este estudo deixou clara a importância de se aprofundar o estudo sobre o bem-estar subjetivo e suas implicações nos diversos âmbitos do ser humano, como trabalho, lazer e educação. Dentre os estudos apontados, houve estudos onde ocorreram correlações entre tais dimensões e o bemestar subjetivo, mas apenas no item otimismo. Neste presente estudo, foi utilizado o bem-estar subjetivo "como um todo" através da escala de afetos positivos e negativos de Zanon (EAZ), versão de pesquisa, e não houve correlação entre o bem-estar subjetivo e o desempenho acadêmico, mensurado pelas médias globais de dois semestres consecutivos, envolvendo dois cursos universitários. 
A importância deste estudo está na evidência de maiores estudos sobre o assunto, que é atual e aponta para alguns índices relevantes, como o otimismo. Com relação ao otimismo especificamente, este é um item do bem-estar subjetivo, e se mostra nas outras pesquisas, correlacionado com o desempenho no trabalho. Nas questões acadêmicas, entretanto, não houve correlação da escala geral de afetos positivos, nem negativos, o que mostra a necessidade de maiores estudos, além do aprofundamento nas questões que envolvem o bem-estar subjetivo, mas não necessariamente a desempenhos e produtividade direta.

Em acréscimo, o presente estudo teve como preocupação principal a preparação do jovem universitário para atuar e "existir" de forma saudável e funcional no contexto laboral. Para tanto, há de se considerar habilidades adicionais que aquelas oferecidas pela formação acadêmica que essencialmente capacita para uma atuação técnica especializada, algo que ainda precisa ser mais bem estudado, conhecido, e oferecido ao estudante universitário.

\section{REFERÊNCIAS}

CONTRERAS, F.; ESGUERRA, G. Psicologia positiva: una nueva perspectiva en Psicología. Diversitas, Universidad Santo Tomás, Bogotá, Colombia, v. 2, n. 2, p. 311-319, jul./dez. 2006.
DELA COLETA, J. A.; DELA COLETA, M. F. Felicidade, bem-estar subjetivo e comportamento acadêmico de estudantes universitários. Psicol. estud., Maringá, v. 11, n. 3, dez.. 2006 .

GARCÍA J. B. D.; SABATÉ J. M. F.; MAZAGATOS V. B. Las Emociones y la dirección ¿ condicinan los afectos de los directivos sus decisiones estratégicas? Universia Business Review, Recoletos Grupo de Comunicación S.A. Madrid, Espanha, n. 17, p. 26-39, jan./mar. 2008.

GIACOMONI, C.H. Bem-Estar subjetivo infantil: conceito de felicidade e construção de instrumentos para avaliação. 2002.Tese (Doutorado).- Universidade Federal do Rio Grande do Sul, Porto Alegre, RS.. Disponível em:

<https://www.repositorioceme.ufrgs.br/han dle/10183/3158>. Acessos em: 24.jun.2013.

GONDIM, S. M. G. et al. Status Profissional e Gênero na Atribuição Intercultural de Afetos no Trabalho. RAM - Revista de Administração Mackenzie, São Paulo, SP, v. 10, n. 4, p. 75-99, jul./ago. 2009.

LONDOÑO PÉREZ, C. Optimismo y salud positiva como predictores de la adaptación a la vida universitaria. Acta Colombiana de Psicologia. Colombia, v. 12 , n. 1, p. 95-107, maio 2009.

MARTINEZ, A. L. Otimismo e Viés de Seleção dos Analistas. BBR - Brazilian Business Review, Vitória, ES,, v. 4, n. 2, p. 104-118, maio/ago. 2007.

MONTEIRO, S. O. M.; TAVARES, J. P. C.; PEREIRA, A. M. S. Optimismo disposicional, sintomatologia psicopatológica, bem-estar e rendimento acadêmico em estudantes do primeiro ano do ensino superior. Estudos de Psicologia, v. 13, n. 1, p. 23-29, 2008.

NERI, A.L. Bienestar Subjetivo en La Vida Adulta y en La Vejez: Hacia Una Psicología 
Positiva en América Latina. Revista Latinoamericana de Psicologia, Fundación Universitaria Konrad Lorenz, Bogotá, Colombia, v.34, n.1-2, p. 55-74, 2002.

VERA NORIEGA J.A.; SOTELO QUIÑONES T.I.; DOMÍNGUEZ GUEDEA M.T. Bienestar subjetivo, enfrentamiento y Redes de Apoyo Social em adultos mayores. Revista Intercontinental de Psicología y Educación, Distrito Federal, México, v.7, n. 2, p. 57-78, jul./dez. 2005.

NORONHA, A. P. P.; MANSÃO, C. S. M. Interesses Profissionais e afetos positivos e negativos: estudo exploratório com estudantes de ensino médio. Psico-USF, Bragança Paulista, v.17, n.2, p. 323-331, maio/ago. 2012.

OTTA, E.; FIQUER, J.T. Bem-estar subjetivo e regulação de emoções. Psicologia em Revista, Belo Horizonte, v.10, n.15, p.144149, jun. 2004. Disponível em: <http://pucmg.br/imagedb/documento/DOC DSC NOME ARQUI20041213115707.pdf>.

Acessos em: 24 jun.2013.

QUEROZ, N. C.; NERI, A. Bem-Estar psicológico e inteligência emocional entre homens e mulheres na meia-idade e na velhice. Psicologia: Reflexão e Crítica, v.18, n. 2, p. 292-299. 2005. Disponível em: <http://www.scielo.br/pdf/prc/v18n2/27481. pdf $>$ Acessos em: 24 jun. 2013.

RAMOS, J. P. ;RAMOS A. M. Q. P. Novas perspectivas da psicologia positiva. Boletim Academia Paulista de Psicologia. Academia Paulista de Psicologia, São Paulo, v. 24, n. 1, p. 57-60, jan./abr. 2004.

SELIGMAN, M.E.P. Felicidade autêntica: usando a nova psicologia positiva para a realização permanente. Rio de Janeiro, RJ: Objetiva, 2004. 